\title{
A experiência de transição para a fase clínica de alunos de medicina detentores de grau prévio: um estudo de caso
}

\author{
Luís Henriques, Ana Salgueira, Nuno Sousa, Manuel J. Costa
}

Introdução. Internacionalmente tem-se assistido à extensão da oferta formativa de cursos de Medicina de menor duração a candidatos detentores de um grau acadêmico superior prévio. Este estudo de caso procura compreender a experiência dos estudantes licenciados nestes cursos, a fim de identificar fatores que condicionem a sua formação durante o inicio da aprendizagem clínica em contexto hospitalar.

Sujeitos e métodos. Os participantes são estudantes licenciados do curso de medicina da Universidade do Minho em Portugal $(n=5)$ que atravessam a transição entre a fase pré-clínica e a fase clínica do curso. No final da primeira unidade curricular clínica realizou-se um grupo de discussão. Os transcritos foram analisados segundo os princípios de Grounded-Theory.

Resultados. Os participantes relataram facilidade no contacto com os pacientes e utilização de competências de estudo durante a aprendizagem. Apontaram como dificuldades principais o primeiro contacto com a morte e a doença no meio hospitalar, a quantidade de conhecimentos a adquirir na antes de iniciarem a formação clínica e a transferência dos mesmos para a prática clínica.

Conclusões. Este estudo de caso revelou que as principais dificuldades dos estudantes licenciados se relacionavam com lidar com pacientes e com a mobilização para a prática da grande quantidade de conteúdo aprendido na fase pré-clínica. Estas dificuldades poderão ser minoradas pela inclusão de maior contacto com pacientes e com a prática clínica na fase pré-clínica.

Palavras chave. Conhecimento médico. Curriculum. Educação médica. Grupo de discussão. Perceção. Residência clínica.

The experience of graduate entry students in the transition to the clinical phase: a case study

Introduction. Internationally, medical schools have been offering more fast-track undergraduate medical degree to graduate applicants. This case study aims to understand graduate entry students' experience in medical schools, namely to identify factors that condition their transition to the clinical training in hospitals.

Subjects and methods. Participants are medical graduate students from the University of Minho in Portugal $(n=5)$ going through the transition from a pre-clinical to a clinical part of a 4 year graduate entry curriculum. A focus group was conducted at the end of the first clinical course. The discussion was transcribed and analyzed using Grounded-Theory principles.

Results. Participants described they were comfortable with contacting patients, and that they applied study skills developed prior to entry medical school to their learning. The main difficulties pointed out their were the first contact with death and disease in the hospital environment, the large amount of content to be learned before starting clinical training, and the transfer of knowledge to clinical practice.

Conclusions. This case study revealed that the main difficulties presented by medical graduate-entry students were related to the first contacts with patients and the practical application of a huge amount of knowledge, learned during the preclinical part of the curriculum, to the clinical practice. These difficulties could be attenuated by including more contact with patients and clinical practice during the pre-clinical part of the course.

Key words. Clinical clerkship. Curriculum. Focus groups. Health knowledge. Medical education. Perception.

Escola de Ciências da Saúde. Universidade do Minho. Braga, Portugal.

Correspondencia: Prof. Luís Henriques. Escola de Ciências da Saúde. Universidade do Minho. Campus de Gualtar. 4710-057 Braga, Portugal.

E-mail: luish88@hotmail.com

Conflicto de intereses: No declarado.

Competing interests: None declared.

(c) 2014 FEM 


\section{Introdução}

A implementação do processo de Bolonha na Europa tem questionado a estrutura tradicional dos cursos de medicina, designadamente os seus objectivos, conteúdos e as suas práticas pedagógicas [1]. Nesse sentido têm tido lugar desenvolvimentos internacionais incluindo Espanha e Portugal [2] sobre, por exemplo, a estruturação de cursos em função das competências do médico [2], a implementação de estruturas curriculares em 2 ciclos [3].

Paralelamente aos desenvolvimentos de Bolonha, a Europa toma consciência da importância de admitir estudantes com licenciaturas prévias aos cursos do ensino superior. Com efeito, muitas escolas de medicina em vários países como a Austrália, Reino Unido [4,5] e também Portugal [6], têm estendido a sua oferta a este novo grupo de estudantes, muitas vezes criando currículos adaptados.

No Reino Unido, o tema da diversificação da população estudantil é particularmente relevante sendo promovido explicitamente na iniciativa Widening Access [7], que visa aumentar a diversidade social e reduzir o elitismo na população de estudantes de medicina, criando oportunidades de acesso a grupos sociais tradicionalmente excluídos [8,9]. As expectativas institucionais relativas aos estudantes adultos é que possuam uma capacidade de aprendizagem superior aos jovens que acabam de terminar o ensino secundário, decorrentes da sua maior maturidade, sentido de responsabilidade e auto-motivação e da sua experiência prévia de sucesso no ensino superior.

Estudos em curso concluíram que os estudantes licenciados têm características específicas, descrevendo-os como sendo menos ansiosos, confiantes, controlados, indagadores e detentores de uma maior maturidade emocional quando comparados com os estudantes provenientes diretamente do ensino secundário $[10,11]$.

Estudos comparativos das experiências enquanto estudantes de medicina, revelam que os estudantes licenciados apresentam desempenhos académicos idênticos aos estudantes tradicionais $[12,13]$ ou até superiores [14]. Os estudantes licenciados demonstram também uma maior segurança e motivação quanto à sua escolha profissional, [15]. Ao nível da selecção dos estudantes, autores como [16] Ian Blackman confirmam a relação entre um percurso académico prévio em áreas científicas e um melhor desempenho académico no curso de medicina. Outros autores relatam não existir uma desvantagem significativa por parte dos estudantes licenciados provenientes de outras áreas $[13,17]$.
A redução de seis para quatro anos de cursos de medicina, tem sido um modelo explorado com a finalidade de os adequar à população de estudantes adultos com um grau académico superior. Trata-se de um modelo usado com sucesso internacionalmente que pressupõe que os conhecimentos e as competências desenvolvidas durante a formação superior prévia dos candidatos, lhes conferem as características necessárias e suficientes para iniciarem a sua formação médica. Estes programas com duração reduzida foram pioneiros na Austrália, iniciados em 1997, e têm vindo a generalizar-se na Europa [18].

No caso específico de Portugal, duas universidades oferecem actualmente um curso de medicina para alunos licenciados com a duração de quatro anos seguindo modelos distintos. O programa da Universidade do Algarve, instituído em 2009, é baseado no modelo tradicional de problem based learning (PBL), no qual o ensino das ciências básicas e clínicas decorre essencialmente através da exploração de casos que os estudantes devem trabalhar autonomamente em pequenos grupos [19]. Na universidade do Minho, é oferecido o modelo de quatro anos como percurso alternativo ao curso de seis anos.

Em Portugal, os estudantes do percurso de quatro anos são também uma população característica, sendo mais velhos, vindos de meios socioeconómicos mais desfavorecidos, e estando mais predispostos a trabalhar em pequenas cidades, quando comparados com os outros [20].

A transição do estudante de medicina de uma fase pré-clínica para uma fase clínica do curso, é um ponto-chave e um dos mais stressantes na preparação da aprendizagem clínica dos estudantes [21]. Trata-se de um período particular, ao requerer que o estudante descubra a sua identidade profissional de médico em contacto com os doentes [22] aplicando conhecimentos teóricos pela primeira vez na prática clínica. Trata-se de uma adaptação a um novo contexto de aprendizagem, a novas formas de ensino e aprendizagem. Relativamente à transição para a clínica dos estudantes licenciados os escassos estudos existentes concluem que as dificuldades encontradas são comparáveis às encontradas pelos estudantes de percursos mais longos [23].

Este artigo pretende corresponder à necessidade de compreender a experiência dos estudantes licenciados durante o período de transição de uma fase pré-clínica para uma fase clínica num curso com um plano de estudos de quatro anos numa escola médica portuguesa. O objectivo foi apurar através de uma discussão de grupo exploratória, que desa- 
fios e que obstáculos estes estudantes sentem durante o mencionado período e qual o contributo da sua formação prévia para a superação ou afirmação dos desafios encontrados.

\section{Sujeitos e métodos}

\section{Contexto do estudo}

Este estudo exploratório foi conduzido na Escola de Ciências da Saúde da Universidade do Minho (ECSUM), em Portugal. O curso de medicina com mestrado integrado da ECS-UM inclui um percurso de 6 anos destinados a estudantes admitidos pelo Concurso Nacional de Acesso ao Ensino Superior, ao qual se candidatam maioritariamente estudantes oriundos do ensino secundário, e um 'percurso alternativo' de quatro anos para estudantes detentores de um grau académico prévio.

Os candidatos às 18 vagas anuais do percurso alternativo são selecionados através de um teste escrito que engloba as disciplinas de biologia, química, física e matemática um em conjunto com processo de mini entrevistas [6,24]. Não é feita qualquer restrição em termos de formação superior prévia. Os candidatos admitidos no ano letivo a que se refere este trabalho detinham as seguintes licenciaturas: Análises Clínicas; Anatomia e Citologia Patológica; Biologia; Genética e Microbiologia; Bioquímica; Cardiopneumologia; Enfermagem; Engenharia Biológica; Farmácia; Físico-Química; Medicina Dentária; Eletrónica e Engenharia Industrial; Química; Radiologia.

Os estudantes admitidos frequentam, no primeiro ano do seu curso, uma unidade curricular denominada 'Fundamentos de Medicina' que pretende habilitar os estudantes com os meios de aquisição de conhecimentos científicos, desempenhos e atitudes nas áreas de anatomia, fisiologia, histologia, embriologia e bioquímica ainda nas áreas de patologia, genética, imunologia, microbiologia/parasitologia e farmacologia, de forma integrada e coordenada, identificando a sua importância na prática médica.

A unidade curricular 'Fundamentos de Medicina' é composta por 6 módulos: metabolismo; sistema circulatório; sistema respiratório; sistema génito-urinário; infeção e imunidade e sistema locomotor e nervoso, cujos conteúdos compreendem a aprendizagem integrada de objetivos em várias disciplinas. Esta unidade decorre paralelamente a outra denominada 'Saúde Comunitária, Ciências Sociais e Humanas' que pretende dotar os futuros mé- dicos de atitudes e aptidões na compreensão dos determinantes chave de saúde e doença nos indivíduos, dos seus condicionantes familiares e sociais, e do desenvolvimento de uma postura humanizada face ao indivíduo e sua família. Os alunos do percurso alternativo completam o final do seu primeiro ano frequentando, em conjunto com os restantes alunos, a unidade curricular 'Introdução à Medicina Clínica', focada nos fundamentos e prática da entrevista e exame físico. Após a conclusão do seu primeiro ano, são integrados com os alunos do quarto ano do percurso tradicional. As unidades curriculares destes anos têm como objetivo principal a aprendizagem da prática clinica, decorrendo essencialmente em contexto de serviços hospitalares e de cuidados primários.

O contacto inicial com a realidade assistencial dos estudantes do percurso alternativo decorre nas últimas semanas do seu primeiro ano, no âmbito da unidade curricular 'Introdução à Medicina Clinica' (IMC). Esta unidade curricular constitui, por isso, um momento importante em termos motivacionais e académicos, pondo à prova os conhecimentos destes estudantes. Os estudantes do programa de 6 anos frequentam a mesma UC mas têm maior contacto com o ambiente assistencial, pois existem unidades curriculares que promovem gradualmente o contacto com esse ambiente desde o início do curso, determinada pela filosofia de integração curricular em $\mathrm{Z}$ [25].

\section{Método de recollha de dados}

Com a intenção de compreender a experiência dos estudantes licenciados durante a transição de uma fase pré-clínica do curso para uma fase clínica do mesmo, foi conduzida uma discussão de grupo semiestruturada com cinco participantes $(n=5)$.

\section{Participantes}

Visando a compreensão da experiência dos estudantes licenciados durante o mencionado período de transição, foi utilizada uma amostra intencional [26], que se refere à selecção de sujeitos com maior potencial informativo. Como tal, todos os sujeitos eram estudantes licenciados da mesma turma, no mesmo momento formativo, após a conclusão da unidade curricular de Introdução à Medicina Clínica.

Os participantes foram recrutados por correio electrónico, numa mensagem enviada pelo coordenador da unidade de educação médica (MJC) a todos os estudantes do percurso alternativo inscritos no curso $(n=18)$. A mensagem explicava o objecti- 
Tabela I. Perguntas feitas no grupo de discussão.

Como é que estão a experienciar esta nova fase do curso, de introdução à medicina clínica?

Querem fazer algum comentário sobre alguma coisa que tenham ouvido, que não tenham percebido e que não tenham sido vocês os emissores?

Vocês querem focar agora um bocadinho com mais detalhe aquilo que agora acham que são as principais dificuldades que têm a aprender a parte clínica?

Quais são as dificuldades que vocês sentem na aprendizagem clínica, neste momento?

Tendo em conta a vossa preparação prévia, e estou a dizer toda, não estou a restringir-me a nenhum período, o que é que, na vossa opinião, ajudou neste período de transição?

Em que medida ajudou nas dificuldades que mencionaram?

De que forma o vosso percurso pré universidade trouxe elementos que ajudaram?

O que houve na vossa formação prévia que não ajudou nada?

Vocês são diferenciados academicamente relativamente aos estudantes provenientes do secundário, pois são doutorados. Isso trouxe alguma coisa?

Que características e competências não académicas, desenvolvidas durante o doutoramento, ajudaram na transição?

Que outros aspectos, na preparação que vos foi dada, poderiam ser implementados para melhora-la?

O que é que, atendendo à preparação que tiveram durante o primeiro ano, sentiram ou sentem mais falta e que vos pudesse ter preparado melhor?

Considerando aquilo que vocês têm vivido, aquilo que é exigido neste momento, o que é que vocês próprios teriam feito de forma diferente na vossa preparação durante a fase pré-clínica do curso?

O que é que vocês próprios fariam de diferente?

Até agora nenhum de vocês falou na vossa vida pessoal e profissional. De que forma isso ajudou ou não ajudou durante esta transição?

Mais alguma coisa a acrescentar?

vo do estudo e contextualizava a sua importância para a compreensão da experiencia destes estudantes numa perspectiva de identificar aspectos do curso susceptíveis de merecerem modificações.

\section{Elaboração do guião}

O guião foi elaborado por dois autores (MJC e AS). Numa primeira fase, foi realizada uma pesquisa bi- bliográfica sobre dificuldades de estudantes de medicina no momento da transição para a formação eminentemente clínica. A listagem resultante deu origem a um guião, com o total de oito perguntas e respectivos tópicos essenciais, apresentados na tabela I.

\section{Procedimentos}

Tendo em vista a documentação da visão dos estudantes sobre a sua experiência na prática clínica, usou-se um grupo de discussão para identificar temas relevantes ao contexto, reconhecidos a partir do ponto de vista dos estudantes, aproveitando a dinâmica inerente a esta metodologia para incentivar os estudantes a elaborar a sua visão, construindo sobre as ideias uns dos outros, e explorando os motivos subjacentes a eventuais divergências [27].

A discussão de grupo foi moderada por um investigador (MJC) com o auxílio de um anotador (AS). A discussão foi gravada (1 h 25 min) e transcrita verbatim.

Visando uma análise qualitativa dos dados recolhidos, os investigadores consideraram os princípios encontrados em Grounded-Theory [28-30] como sendo os mais apropriados para a sua realização, dado o seu potencial na compreensão do significado das experiências dos sujeitos. Open-coding bem como axial-coding foram utilizados na concretização desta análise.

As transcrições foram codificadas independentemente por dois investigadores (LH e MJC) usando uma análise linha-a-linha para caracterizar os códigos presentes no texto. Os investigadores reuniram-se e discutiram a codificação até chegarem a um consenso quanto aos códigos finais. Analisaram-se pontos em comum entre os vários comentários dos participantes para discernir pontos-chave. Os códigos foram então agrupados.

\section{Resultados}

A reunião dos vários comentários feitos pelos participantes permitiu a conceptualização de duas categorias principais de análise. Uma prende-se com a experiência dos estudantes durante os seus primeiros contactos com os pacientes, e a outra com refere-se às experiências de aplicação prática de conhecimentos em contexto clínico durante a transição. Os comentários mais pertinentes referentes a cada uma e aceites de forma consensual pelos participantes encontram-se presentes nas tabelas relativas a cada categoria. 


\section{Relação estudante/paciente (Tabela II)}

Os participantes relataram alguns desafios relevantes associados ao contacto inicial com a prática clínica em contexto hospitalar. O tema mais salientado foi o impacto da constante presença da morte, dos doentes e da doença no Hospital (amostra 1.a.). Vários participantes se referiram à carga emocional imposta pela necessidade de lidar com a morte dos pacientes e com o constante contacto com os doentes. Este aspeto era considerado dominante, por exemplo, sobre as dificuldades de gestão e aplicação de conhecimento no novo contexto (amostra 1.b). Houve referência ao facto de, terminado o período diário de aprendizagem no Hospital, os estudantes transportarem a vivência da morte para casa (amostra 1.b). Foi evocado o termo 'complexo' (amostra 1.c.) e um participante referiu explicitamente a 'mossa' sentida, revelando assim alguma impreparação emocional para esta experiência. Esta percepção coabita com a noção de que ser mais velho intensificará os desafios emocionais anteriores (amostra 1.c.).

Os participantes relataram também desafios no que se refere à necessidade de explorar um espaço íntimo durante a abordagem a doentes em avançado estado de debilidade física ou emocional. Adicionalmente referiram sensibilidade no que concerne à realização do exame físico ao paciente, em particular nos contactos iniciais. (amostra 1.e.).

Do mesmo modo, os estudantes demonstram uma grande preocupação pelo bem-estar do paciente, conferindo-lhe primazia sobre as necessidades do seu treino de procedimentos técnicos (amostra 1.f.).

Apesar dos desafios encontrados e das dúvidas aquando o seu primeiro contacto com a prática clínica, os participantes denotaram estarem atentos à sua postura profissional perante os doentes (amostra 1.g).

Em relação aos estudantes do percurso de 6 anos, os estudantes licenciados relatam facilidade (amostra 1.h.) e mesmo algumas vantagens no que se refere à comunicação e abordagem aos pacientes. Parte dessas vantagens referem-se à sua compreensão da linguagem utilizada por pacientes mais velhos (amostra 1.i.) e à sua tomada de iniciativa perante situações sensíveis (amostra 1.j.).

Estes estudantes relataram um grande nível de satisfação pelo seu contacto com outras pessoas, doentes ou não, durante a sua aprendizagem (amostra 1.k.).

\section{Aplicação de conhecimentos (Tabela III)}

Ao pronunciar-se sobre o contributo da sua formação durante o primeiro ano no curso de medicina
Tabela II. Amostras referentes às primeiras abordagens aos pacientes.

1.a. '[...] foi bastante complexo para mim lidar com a morte e com a doença'

1.b. 'A parte mais complexa para mim foi conviver diariamente com a morte e com a doença, e essas segundas partes, portanto o ver se o que tinha aprendido servia para alguma coisa ou era suficiente, a integração com os colegas, no meu caso, acabou por ser secundária a nível de impacto pessoal. Ou seja, eu ia para casa a pensar, não no se me dei bem com os colegas, não sobre o ser suficiente para estar aqui, mas ia para casa a pensar no que tinha visto'

1.c. 'E por acaso acho que nos faz mais mossa, estranhamente. Acho que para alguém de 20 anos ou 19 anos a morte ainda é demasiado distante e não a sente da mesma forma'

1.d. '[...] eu não sabia o que fazer, porque eu estava com o coração e... estava mesmo emocionada de ver a senhora também ali'

1.e. 'Eu senti algumas dificuldades porque acho que tudo é tão privado, é tanta a privacidade da pessoa, muitas vezes no estado de debilidade emocional e física em que a pessoa se encontra que me custou as primeiras abordagens ao exame físico'

1.f. '[...] e a minha preocupação obviamente não era estar ali com as coisas técnicas, era mesmo o respeito e ter a capacidade de perceber até onde é que eu poderia ou não magoar o doente'

1.g. 'Mesmo nós tendo os mesmos receios que eles [estudantes do percurso tradicional] sabíamos enfrentar a situação de uma forma profissional'

1.h. 'A abordagem aos doentes não foi problema nenhum'

1.i. 'Mas é natural. Até mesmo em questões de nomenclatura, palavras que os do percurso normal [...] como por exemplo «jornaleiro», às vezes o diálogo com o doente [...]. Eu lembro-me de estar a traduzir para as minhas colegas o que ele estava dizer'

1.j. '[...] é desconfortável, de facto, estar ali a entrar na intimidade, mas assumo isso de uma forma natural. Os meus colegas não. Ficavam constrangidos e tinha que eu dar o avançar com o passo'

1.k. 'Por outro lado a parte de lidar com pessoas apesar de ser complicada, acho que para mim foi das experiências mais positivas do ano todo'

para a sua experiência clínica, os estudantes destacaram três aspetos fundamentais:

- Dificuldades na aplicação, perante o doente, de conhecimentos adquiridos em contexto académico.

- A perceção de estarem equiparáveis aos colegas do percurso de 6 anos no que se refere à sua preparação teórica.

- A importância do contacto com a prática clínica, percecionada a partir das suas próprias vivências ou inferida a partir de docentes clínicos, como essencial para a estruturação das suas aprendizagens.

Um dos principais desafios relatados está relacionado com a ignorância da forma como os conhecimentos académicos são reorganizados para terem utilidade prática. Daqui resultaram expressões de receio de falta de preparação para a aplicação dos conhecimentos (amostra 2.a.), independente da sua preparação teórica (amostra 2.b.). Esta mobilização 
Tabela III. Amostras referentes à aplicação de conhecimentos em contexto clínico.

2.a. 'Aquilo que eu senti mais [...] era saber se de facto eu estou preparada em termos de teoria para poder na parte prática aplicar aquilo que eu sei'

2.b. 'Mas o conhecimento não é tão facilmente mobilizável para a realidade clínica [...]. A partir daí essa mobilização para mim é aquilo que eu tenho alguma dificuldade, não porque não tenho os conhecimentos [... $]^{\prime}$

2.c. '[...] senti-me absolutamente ao nível dos outros colegas [estudantes do percurso tradicional]'

2.d. 'Aquilo que eu reparei é que em termos teóricos não estamos assim tão diferentes em relação aos outros colegas'

2.e. 'Por isso é que eu volto a insistir que, pelo menos no meu caso é importante ter tempo para sedimentar os conteúdos'

2.f. 'O que estaria pior é não termos tempo de amadurecer aquilo que nós aprendemos'

2.g. 'É a questão de amadurecer os conhecimentos, e quanto mais abordamos mais memorizamos, não é?'

2.h. ' $[\ldots]$ porque realmente não tive tempo para sedimentar os meus conhecimentos, e ver e rever...'

2.i. 'A matéria que se dá num dia, no dia a seguir, as horas da manhã não são suficientes para estudar, a maior parte das vezes, uma aula sequer, quanto mais a matéria que se deu inteira de um dia'

2.j. 'Depois não tinhas tempo para estudar, o problema era esse'

2.k. '[...] a decisão é «o que é que vai ficar de fora? O que é que não vou estudar desta vez?»'

2.I. '[A experiência clínica] ajudou-me certamente muito para no próximo ano ter já uma base muito mais sólida para no fundo adquirir os conhecimentos e até saber já como. Como já tenho a perspectiva de como depois mobilizar para a parte da prática clínica, vou fazer já uma abordagem diferente'

2.m. 'Como já tive a experiência da parte clínica, daqui para a frente sei onde estão as minhas falhas, o que é que tenho que estudar mais, o que não tenho e de certa forma isso ajuda'

2.n. '[...] como temos o tempo limitado, restrito, se calhar era mais vantajoso termos dedicado esse tempo a outros assuntos mais importantes ou com um grau de dificuldade maior e que houvesse mais necessidade de explorar'

2.o. 'Se calhar também diria, [um doutoramento ajuda a] saber distinguir o que é importante do que não é importante e do que é acessório. Porque tu na licenciatura não tens essa prática'

2.p. '[... ] se as aulas fossem abordadas mais por clínicos e não por pessoas de cada uma das especialidades das ciências básicas, que se calhar nos beneficiaria, lá está, naquele sentido de a gente perceber se aquilo é ou não é tão importante que justifique a gente dedicar algumas horas de estudo [...]'

2.q. '[...] nós temos o tempo muito limitado e à partida a parte de fundamentos seria para abordar aquilo que realmente era mais importante e que iria ser mais rotineiro'

2.r. 'Eu acho que teve uma lacuna muito grande, que foi precisamente nós não termos tido mais componente prática, e para treinar estes procedimentos que nós temos que fazer em seres humanos [... $]^{\prime}$

de conhecimentos para a prática clínica é um processo distinto do da aquisição dos conhecimentos a serem mobilizados, que também suscita dificuldades particulares, exploradas seguidamente.

Quanto à sua preparação, os estudantes referiram que o ano de aprendizagem de conhecimentos que precedeu a sua experiência em IMC lhes havia conferido um nível de preparação equivalente ao dos estudantes do currículo normal. Apesar dos desafios relativos à aplicação de conhecimentos, e mesmo tendo apenas um ano de aprendizagem préclínica, os estudantes licenciados não sentiram qualquer disparidade quanto ao nível de preparação relativa a conhecimentos teóricos para a fase clínica do curso, afirmando que se sentem tão bem preparados como os estudantes do currículo tradicional (amostras 2.c. e 2.d.).

Apesar do seu sentimento de igual preparação teórica quando comparados com os estudantes do percurso tradicional, os estudantes licenciados descrevem uma falta de 'amadurecimento' ou consolidação dos conhecimentos. Os participantes consideraram que o período de aprendizagem era insuficiente para proporcionar as circunstâncias necessárias a esse 'amadurecimento' (amostras 2.e.; 2.f.; 2.h.). O outro aspeto evocado foi a inexistência de oportunidades suficientes para a prática dos conhecimentos adquiridos (amostras 2.g.; 2.h. e 2.r.),

Foram referidas outras condicionantes associadas ao fator 'insuficiência de tempo', por exemplo, como um importante condicionante do tempo disponível para estudar e, por conseguinte, como um forte obstáculo ao desenvolvimento do seu conhecimento (amostras 2.i. e 2.j.).

Os dados parecem apontar para o facto de um grande volume de informação e conhecimentos a adquirir, conciliado com o curto espaço de tempo que têm, levar a um sentimento de desorientação, conduzindo os estudantes a reconsiderarem as suas estratégias de estudo, reestruturando e priorizando os conhecimentos a adquirir (amostras 2.k.; 2.n.; 2.q.). Esta priorização, imposta pelas suas dificuldades, foi uma das maiores preocupações revelada pelos participantes.

Neste sentido, a vivência clinica tem efeitos sobre a forma com os estudantes consideram abordar o estudo, sugerindo que um contacto precoce com a prática clínica seria benéfica para a sua abordagem desde o início do curso. Vários fatores parecem contribuir para esta priorização dos conteúdos a aprender. Entre os principais, destaca-se o papel da experiência clínica (amostras 2.l. e 2.m.), que revela também ter um papel relevante na abordagem ao estudo e na preferência dos estudantes por docentes experientes na prática clínica (amostra 2.p.).

Outros fatores adicionais sugeridos como possíveis agentes nesta priorização de conhecimentos a adquirir são o grau de dificuldade dos conhecimentos (amostra 2.n.), levando os estudantes a darem prioridade ao estudo de matérias que considerem 
mais difíceis, e a perceção que têm da frequência com que vão aplicar esses conhecimentos na prática clínica (amostra 2.q.), sendo que os estudantes relatam dar prioridade à aprendizagem de conhecimentos que prevejam aplicar mais frequentemente. A priorização de conteúdos apresentada por docentes clínicos é referida também como um fator relevante na priorização das aprendizagens (amostra 2.p.), assim como o seu próprio juízo e competências de estudo provenientes da sua experiência enquanto estudantes autónomos durante o seu grau académico prévio (amostra 2.o.).

\section{Discussão}

É necessário conhecer e compreender a experiência dos estudantes nos momentos de transição dos seus cursos de medicina para identificar pontos onde introduzir melhorias, tenham estas a ver com a sua aprendizagem a longo prazo ou com o melhoramento da experiência nesses momentos particulares. Tal torna-se particularmente importante no caso dos estudantes admitidos com o grau de licenciado em cursos de menor duração, tendo em consideração que se trata de um modelo formativo mais recente. Este estudo de caso, baseado numa entrevista a estudantes pouco tempo após a sua transição para a parte predominantemente clínica de um curso, revelou que os aspetos mais problemáticos se prendiam com lidar com aspetos humanos da atividade médica e com a mobilização de conhecimentos adquiridos anteriormente de forma a poderem ser usados com eficácia nas interações sem contexto clínico.

Os elementos extraídos da análise qualitativa revelaram que os estudantes licenciados consideram estar em situação de vantagem num conjunto de circunstâncias. No que respeita à relação médicodoente, é notória a sua preocupação com o bemestar dos pacientes e aforma como privilegiam o bem-estar do paciente sobre as necessidades que estes estudantes apresentam de praticar procedimentos clínicos. Ao mesmo tempo, relativamente aos seus colegas admitidos diretamente do ensino secundário, consideram ter maior iniciativa para lidar com situações delicadas, mais facilidade que os estudantes do currículo tradicional no estabelecimento da relação com os pacientes, na compreensão do seu discurso, bem como um enorme gosto pelo contacto com os mesmos.

Relativamente à aprendizagem de conhecimentos, a entrevista não permitiu identificar benefícios provenientes da sua formação académica anterior.
Os resultados sugerem que o curso de medicina solicita aos estudantes o conhecimento de uma forma diferente daquela com que organizaram os seus conhecimentos académicos até ao momento. Ainda assim, foi possível concluir que as competências genéricas de estudo são consideradas relevantes para a aprendizagem. Segundo a perspetiva dos estudantes entrevistados, frequentar um curso de medicina com um curso prévio é vantajoso por tirarem partido das competências de estudo adquiridas durante a sua formação anterior.

No que se refere às dificuldades identificadas pelos estudantes licenciados durante os seus primeiros contactos com a prática clínica, uma das mais prementes advém do choque do primeiro contacto destes estudantes com o meio hospitalar, e refere-se à constante presença e convivência com a morte, com a doença e com os doentes. A idade dos estudantes foi apresentada como um fator significativo na afirmação desta dificuldade (amostra 1.a.).

Ao mesmo tempo, os participantes do grupo de discussão referiram ter algumas dificuldades na aplicação na prática clínica de conhecimentos adquiridos na unidade curricular precedente, tendo mencionado especificamente que tal dificuldade não advém da falta de conhecimentos (amostra 2.b.).

A falta de tempo relatada por estes estudantes aparenta ter um impacto negativo no seu nível de preparação para a prática clínica. Embora estes estudantes relatem um nível de preparação idêntico ao dos estudantes do percurso tradicional, o tempo é apresentado como um fator importante para a integração dos conhecimentos. Em conjunto com a falta de repetição das aprendizagens, (amostras 2.g. e 2.h.), estes dois fatores parecem dominar as dificuldades encontradas por estes estudantes no que se refere à sua preparação teórica. Embora não tenham sido relatadas dificuldades quanto à assimilação de novos conhecimentos, os comentários relatam problemas no que se refere à acomodação dos mesmos, mais especificamente à relação da quantidade de conhecimentos a adquirir com a curta duração do período pré-clínico, sugerindo dificuldades evidenciadas pela teoria da carga cognitiva [31]. Esta teoria explica que o esforço exercido sobre a memória de trabalho de um sujeito quando este tenta adquirir novos conhecimentos, depende da quantidade de informação a adquirir, o tempo que tem para o fazer, e das estruturas de conhecimento que detém. Neste sentido, um especialista numa área terá mais facilidade em processar uma grande quantidade de informação nova num curto espaço de tempo pois já possui estruturas de conhecimentos adequadas à sua integração, por contraste às es- 
truturas de conhecimento detidas por um principiante. Os dados sugerem que as dificuldades apresentadas pelos estudantes licenciados se referem à falta de estruturas de conhecimento em medicina, o que os impede de adquirir tanta informação em tão pouco tempo.

Segundo os participantes, esta dificuldade força os estudantes a selecionar os conhecimentos a adquirir. Neste sentido, a experiência clínica parece ter um papel fulcral enquanto orientadora do estudo. A previsão da frequência com que vão aplicar determinados conhecimentos, bem como a previsão do impacto que as suas práticas poderão ter no bem-estar dos pacientes, aparentam ser fatores determinantes quando estes estudantes se vêm obrigados a escolher o que estudar. Estas preocupações refletem-se no facto de preferirem aulas dadas por docentes com experiência na prática clínica.

Os resultados deste estudo têm uma aplicabilidade prática no que se refere à estruturação dos cursos de medicina de duração reduzida para estudantes licenciados. Para além de confirmar que a experiência dos estudantes licenciados lhes pode conferir vantagens -essencialmente na relação médico-doente e nas competências genéricas de estudo.

Os resultados sugerem a conveniência de proporcionar a estes estudantes mais prática clínica na fase inicial da formação dos estudantes licenciados. Neste sentido, será útil considerar a adoção de uma estrutura curricular atendendo a uma integração vertical em forma de Z [25], incorporando o ensino das ciências biomédicas com a aprendizagem clínica desde o início do curso. Atendendo aos resultados, esta integração ajudaria a atenuar o impacto do primeiro contacto dos estudantes com a realidade no hospital, e teria um papel fulcral enquanto orientadora do estudo e reorganizadora do processo de aprendizagem.

Do mesmo modo, a falta de tempo relatada, a grande quantidade de conhecimentos a adquirir, e as dificuldades que dela advém, poderão ter implicações na discussão sobre a duração dos cursos de medicina para estudantes licenciados. Apesar das competências de estudo relatadas por estes estudantes, as limitações e o processo de desenvolvimento apresentados pela teoria da carga cognitiva deverão ser tomadas em conta durante a estruturação destes cursos de duração reduzida.

Embora este estudo de caso apresente limitações relativas ao tamanho da amostra e ao seu foco de análise, acrescenta novos elementos sobre a experiência dos estudantes licenciados nos cursos de medicina, e poderá contribuir para a orientação de estudos futuros sobre este assunto.
Em conclusão, uma análise qualitativa sobre a experiência dos estudantes licenciados evidencia dificuldades características deste grupo de estudantes, mas também perceções de vantagens relativas aos seus colegas admitidos diretamente do ensino secundário.

Bibliografia

1. Palés J. El proceso de Bolonia más allá de los cambios estructurales. Una visión desde la educación médica en España. REDU: Revista de Docencia Universitária 2012; 10: 35-53.

2. Patricio M, Palés J. El marco en el que nos movemos: la educación médica en Europa y la perspectiva desde el ámbito ibérico. Espacio Europeo de Educación Superior 2008; 1: 18-25.

3. Patricio M, De Burbure C, Costa MJ, Schirlo C, Ten Cate O. Bologna in medicine anno 2012: experiences of European medical schools that implemented a Bologna two-cycle curriculum -an AMEE-MEDINE2 survey. Med Teach 2012; 34: 821-32.

4. Carter YH, Peile E. Graduate entry medicine: high aspirations at birth. Clin Med 2007; 7: 143-7.

5. Howe A, Campion P, Searle J, Smith H. New perspectives: approaches to medical education at four new UK medical schools. BMJ 2004; 329: 327-31.

6. Marvão P, Ponte J. Mini-entrevistas múltiplas: um método de seleção de estudantes para o ensino superior. XII Congresso Galego-Português de Psicopedagogia. Braga: Universidade do Minho; 2013.

7. Universities UK. Social class and participation: good practice in widening access to higher education. London: UK; 2002.

8. Powis DA, Hamilton J, McManus IC. Widening access by changing the criteria for selecting medical students. Teaching and Teacher Education 2007; 23: 1235-45.

9. James D, Ferguson E, Powis D, Symonds I, Yates J. Graduate entry to medicine: widening academic and socio-demographic access. Med Educ 2008; 42: 294-300.

10. James D, Ferguson E, Powis D, Bore M, Munro D, Symonds I, et al. Graduate entry to medicine: widening psychological diversity. BMC Med Educ 2009; 9: 67.

11. Rushforth B. Life in the fast lane: graduate entry to medicine. Student BMJ 2004; 12: 368-70.

12. Rolfe IE, Ringland C, Pearson SA. Graduate entry to medical school? Testing some assumptions. Med Educ 2004; 38: 778-86.

13. Shehmar M, Haldane T, Price-Forbes A, Macdougall C, Fraser I, Peterson S, et al. Comparing the performance of graduate-entry and school-leaver medical students. Med Educ 2010; 44: 699-705.

14. Calvert MJ, Ross NM, Freemantle N, Xu Y, Zvauya R, Parle JV. Examination performance of graduate entry medical students compared with mainstream students. J R Soc Med 2009; 102: 425-30.

15. Wilkinson TJ, Wells JE, Bushnell JA. Are differences between graduates and undergraduates in a medical course due to age or prior degree? Med. Educ 2004; 38: 1141-6.

16. Blackman I, Darmawan IG. Graduate-entry medical student variables that predict academic and clinical achievement. Int Educ J 2004; 4: 30-41.

17. Groves MA, Gordon J, Ryan G. Entry tests for graduate medical programs: is it time to re-think? Med J Aust 2007; 186: 120-3.

18. Craig PL, Gordon JJ, Clark RM, Langendyk V. Prior academic background and student performance in assessment in a graduate entry programme. Med Educ 2004; 38: 1164-8.

19. Maudsley G. Do we all mean the same thing by 'problembased learning'? A review of the concepts and a formulation of the ground rules. Acad Med 1999; 74: 178-85.

20. Alves R. O concurso especial para acesso aos cursos de medicina por licenciados introduzem diversidade na população de estudantes de medicina? [in press]. 
21. Radcliffe $C$, Lester $H$. Perceived stress during undergraduate medical training: a qualitative study. Med Educ 2003; 37: 32-8.

22. Dornan T. Experience based learning: learning clinical medicine in workplaces. University of Maastricht: Datawyse; 2006.

23. Garrud P, Yates J. Profiling strugglers in a graduate-entry medicine course at Nottingham: a retrospective case study. BMC Med Educ 2012; 12: 124.

24. Eva KW, Rosenfeld J, Reiter HI, Norman GR. An admissions OSCE: the multiple mini-interview. Med Educ 2004; 38: 314-26.

25. Wijnen-Meijer M, Ten Cate OT, Van der Schaaf M, Borleffs JC. Vertical integration in medical school: effect on the transition to postgraduate training. Med Educ 2010; 44: 272-9.
26. Patton MQ. Qualitative research and evaluation methods. 3 ed. California: Sage Publications; 2002.

27. Barbour RS. Making sense of focus groups. Med Educ 2005; 39: 742-50.

28. Glaser B. Theoretical sensitivity. Mill Valley, CA: Sociology Press; 1976.

29. Glaser B, Strauss A. The discovery of grounded theory. Chicago: Aldine; 1967.

30. Strauss A. Qualitative analysis for the social sciences Cambridge, UK: Oxford University Press; 1988.

31. Paas F, Renkl A, Sweller J. Cognitive load theory: instructional implications of the interaction between information structures and cognitive architecture. Instructional Science 2004; 32: 1-8.

\section{Experiencia de la transición a la fase clínica de los estudiantes de medicina que ingresan con posesión} de otro grado: un estudio de caso

Introducción. En el ámbito internacional se ha extendido la oferta formativa de estudios de medicina de menor duración para candidatos que están en posesión de otros grados de educación superior previos. Este estudio de caso trata de comprender la experiencia de los estudiantes procedentes de otros grados en estos cursos con el fin de identificar los factores que limitan su aprendizaje clínica en el contexto hospitalario.

Sujetos y métodos. Los participantes son estudiantes de posgrado de la Facultad de Medicina de la Universidad de Minho en Portugal $(n=5)$, que realizan la transición entre la fase preclínica y clínica del grado. Al final del primer curso clínico se llevó a cabo un grupo de discusión. Las transcripciones se analizaron de acuerdo con los principios de grounded theory.

Resultados. Los participantes refirieron facilidad para el contacto con los pacientes y para el uso de técnicas de estudio durante su aprendizaje. Las principales dificultades son el primero contacto con la muerte y la enfermedad en el ámbito hospitalario, el volumen de conocimientos que se deben adquirir antes de embarcarse en la formación clínica y su transferencia a la práctica clínica.

Conclusiones. Este estudio de caso reveló que las principales dificultades de los estudiantes procedentes de otros grados se relacionaron con el trato de los pacientes y con la aplicación a la práctica de los numerosos contenidos aprendidos en la fase preclínica. Estas dificultades se pueden reducir mediante un mayor contacto con los pacientes y la práctica clínica en la fase preclínica.

Palabras clave. Aprendizaje clínico. Conocimientos, actitudes y prácticas. Educación médica de pregrado. Grupo focal. Percepción. Plan de estudios. 